CASE REPORT

\title{
Transient osteoporosis of the hip in a non-pregnant woman
}

\author{
Kanakeya Bachha Reddy, Atul Sareen, Rajesh Kumar Kanojia, Jatin Prakash
}

LHMC, Delhi, India

\section{Correspondence to} Dr Jatin Prakash,

jatin_mamc@yahoo.co.in

Accepted 9 May 2015

\begin{abstract}
SUMMARY
Transient osteoporosis of the hip is a rare, self-limiting condition, occurring most commonly in middle-aged men, but also sometimes in women, usually in late pregnancy. It is characterised by gradual onset of hip pain aggravated by weight bearing without any associated history of trauma and systemic illness. It is usually of unknown aetiology, but pregnancy is a recognised risk factor for women. Other conditions that can mimic transient osteoporosis of the hip on MRI are osteonecrosis, osteomyelitis and neoplasms. We present a case of a 38-year-old non-pregnant woman with transient osteoporosis of the hip, managed conservatively, leading to a full recovery. Treatment is conservative, including protected weight bearing, physical therapy and non-steroidal analgesics. The patient was completely painless and symptom free at 2-year follow-up.
\end{abstract}

\section{BACKGROUND}

Transient osteoporosis hip is a rare, self-limiting condition, occurring most commonly in middle-aged men, and also sometimes in women, usually in late pregnancy. ${ }^{1}{ }^{2}$ It is characterised by gradual onset of hip pain aggravated by weight bearing without any associated history of trauma and systemic illness. It is usually of unknown aetiology, but pregnancy is a recognised risk factor for women. Transient osteoporosis of the hip is a selflimiting condition and resolves within 6 months, clinically and radiologically. X-ray changes showing demineralisation of femoral head and neck may not be seen until as long as 6 weeks after the onset of symptoms. MRI shows bone marrow oedema and is highly sensitive for diagnosis of the disorder. ${ }^{3}$ Other conditions that can mimic it on MRI are osteonecrosis, osteomyelitis and neoplasms. ${ }^{4} 5$ Treatment is conservative, including protected weight bearing, physical therapy and non-steroidal analgesics. Only a few cases of transient osteoporosis of the hip in non-pregnant women have been reported in the literature to date. ${ }^{6} 7$ We present a case of a 38-year-old non-pregnant woman with transient osteoporosis of the hip, managed conservatively leading to a full recovery.

\section{CASE PRESENTATION}

A 38-year-old woman presented to us with a 1-month history of pain in the left hip joint. The pain was severe, localised to left hip joint region, aggravated by walking and relieved by resting. The patient had no history of systemic illness and was not taking any medication. There was no history of trauma, morning stiffness, weight loss, anorexia, night cries, skin rashes and fever. She had no history of smoking and alcohol consumption. She has three children, and the last child birth was 12 years ago, at which time bilateral tubal ligation was performed. On examination, her gait was antalgic. There was mild localised tenderness felt on her left groin. At the time of examination all her movements were grossly painful and restricted.

\section{INVESTIGATIONS}

To rule out infection, the patient was investigated. The reports showed haemoglobin $12 \mathrm{~g} \%$, total leucocyte count $5400 / \mathrm{mm}^{3}$ and erythrocyte sedimentation rate (ESR) $25 \mathrm{~mm}$ per first h; C reactive protein (CRP) was negative. X-ray of the pelvis with both hips showed normal findings (figure 1). MRI showed low signal intensity in the femoral head and neck on T1-weighted images (figure 2) and homogeneous high signal intensity on T2-weighted images, with joint effusion and joint space preservation (figure 3). Ultrasound-guided left hip aspiration was carried out and 2-3 mL grossly clear and viscous fluid aspirated. The aspirate was sent for a Gram stain, acid-fast bacilli (AFB) stain, culture and sensitivity, and PCR for Mycobacterium tuberculosis. After $48 \mathrm{~h}$ of culture, no bacterial growth was found, and staining was negative. PCR for M. tuberculosis was also negative.

\section{DIFFERENTIAL DIAGNOSIS}

The differential diagnoses in our case were transient osteoporosis of the hip, avascular necrosis $(\mathrm{AVN})$, septic arthritis, malignancies, inflammatory joint diseases and fracture of the femoral neck. Absence of fever, non-elevated ESR and CRP, and non-purulent arthrocentesis, helped in ruling out septic arthritis. Absence of trauma was against any fracture, as confirmed on X-ray. Monoarticular involvement with no morning stiffness helped in ruling out inflammatory arthritis. However, it was very difficult to differentiate between AVN and transient osteoporosis of the hip. In our patient, the absence of common risk factors for AVN, acute onset, typical MRI findings and no clinical progression supported the transient osteoporosis of the hip diagnosis.

\section{TREATMENT}

Taking all the clinical findings and investigations into consideration, transient osteoporosis of the hip was diagnosed, and the patient was managed conservatively with below knee skin traction and 
Figure 1 X-ray of the pelvis with both hips, anteroposterior and lateral views showing normal radiological findings.

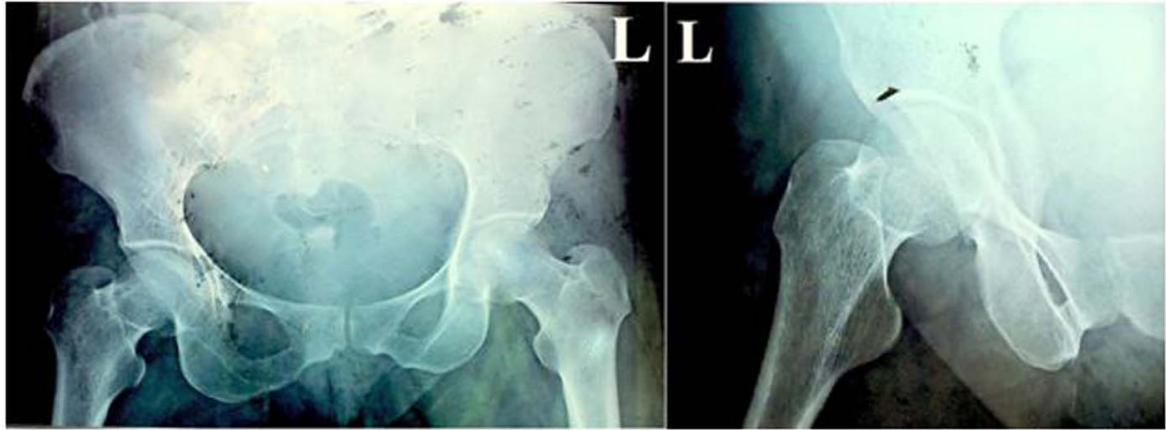

anti-inflammatory drugs for 2 weeks. After 2 weeks, there was symptomatic improvement and the patient was mobilised by walker for non-weight bearing ambulation and active-assisted range of motion exercise for the hip. At 6 weeks of follow-up, repeat X-rays of the pelvis with both hips (anteroposterior and lateral views) were carried out and X-rays confirmed osteoporosis of the left femoral head (figure 4). At 3 months of follow-up, the patient was allowed to bear partial weight on the left lower limb and gradually progressed to full-weight bearing according to pain over a period of 2 months. This benign course and full recovery further supported the diagnosis of transient osteoporosis of the hip in this case.

\section{OUTCOME AND FOLLOW-UP}

The patient is completely painless and symptom free at 2-year follow-up.

\section{DISCUSSION}

Transient osteoporosis of the hip is a rare, self-limiting condition of unknown aetiology, characterised by a sudden onset of joint pain with spontaneous resolution. In 1959, Curtiss and Kincaid described transient osteoporosis of the hip in the third trimester of pregnancy in three women. ${ }^{8}$ Since then, various terms have been used to describe this bone marrow oedema, such as transient osteoporosis of the hip, regional migratory osteoporosis (RMO) and reflex sympathetic dystrophy (RSD). Hoffman et al included all these entities under bone marrow oedema syndrome pattern. ${ }^{9}$ RMO and transient osteoporosis of the hip have been described as common entities. As whether the diagnosis is RMO or transient osteoporosis of the hip, the management remains the same. ${ }^{10}$ Two-thirds of the cases of transient osteoporosis of the hip occur in healthy middle-aged men between the ages of 40 and 60 years, and one-third in women who are in the third trimester of pregnancy. ${ }^{12}$ The hip joint is the most commonly affected are in transient osteoporosis, but it can also affect the knee, foot and ankle. ${ }^{3}$ Differential diagnoses such as AVN, septic arthritis, malignancies, inflammatory joint diseases and fractures of the femoral neck, as well as RSD and pigmented villonodular synovitis, should be taken into consideration. ${ }^{5}$ However, it is very difficult to distinguish between transient osteoporosis of the hip and avascular necrosis of the hip. Rare case reports have documented this pattern presenting as the earliest phase of AVN of the head of the femur. ${ }^{11}$ The recent literature showed that transient osteoporosis of the hip and osteonecrosis are clearly two different entities. ${ }^{12-14}$ Some authors suggested that in transient osteoporosis of the hip, the
Figure $2 \mathrm{MRI}$ of the hip, sagittal and axial cuts showing low intensity in the femoral head and neck on T1-weighted images.

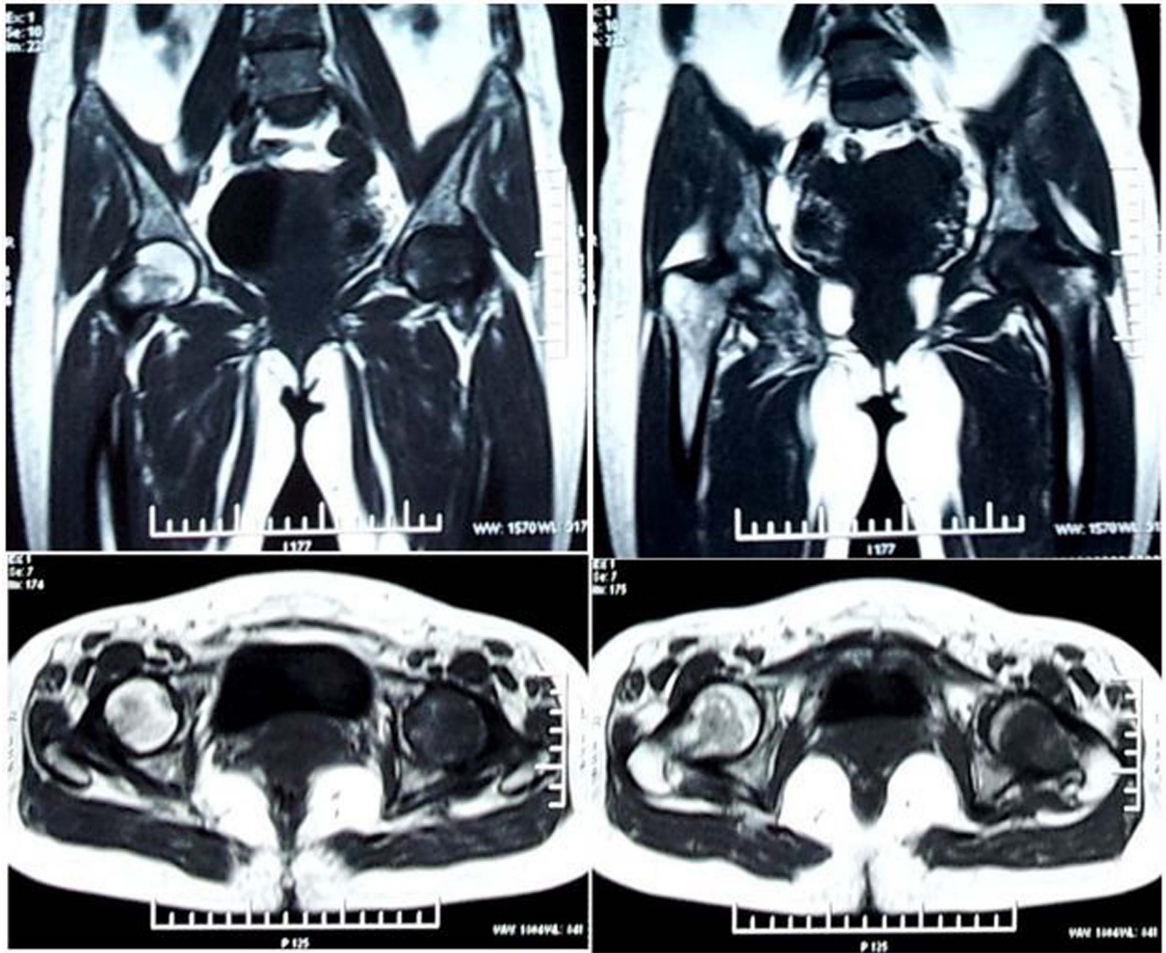


Figure $3 \mathrm{MRI}$ of the hip, sagittal and axial cuts showing high signal intensity in the femoral head and neck on T2-weighted images with joint effusion and joint space preservation.
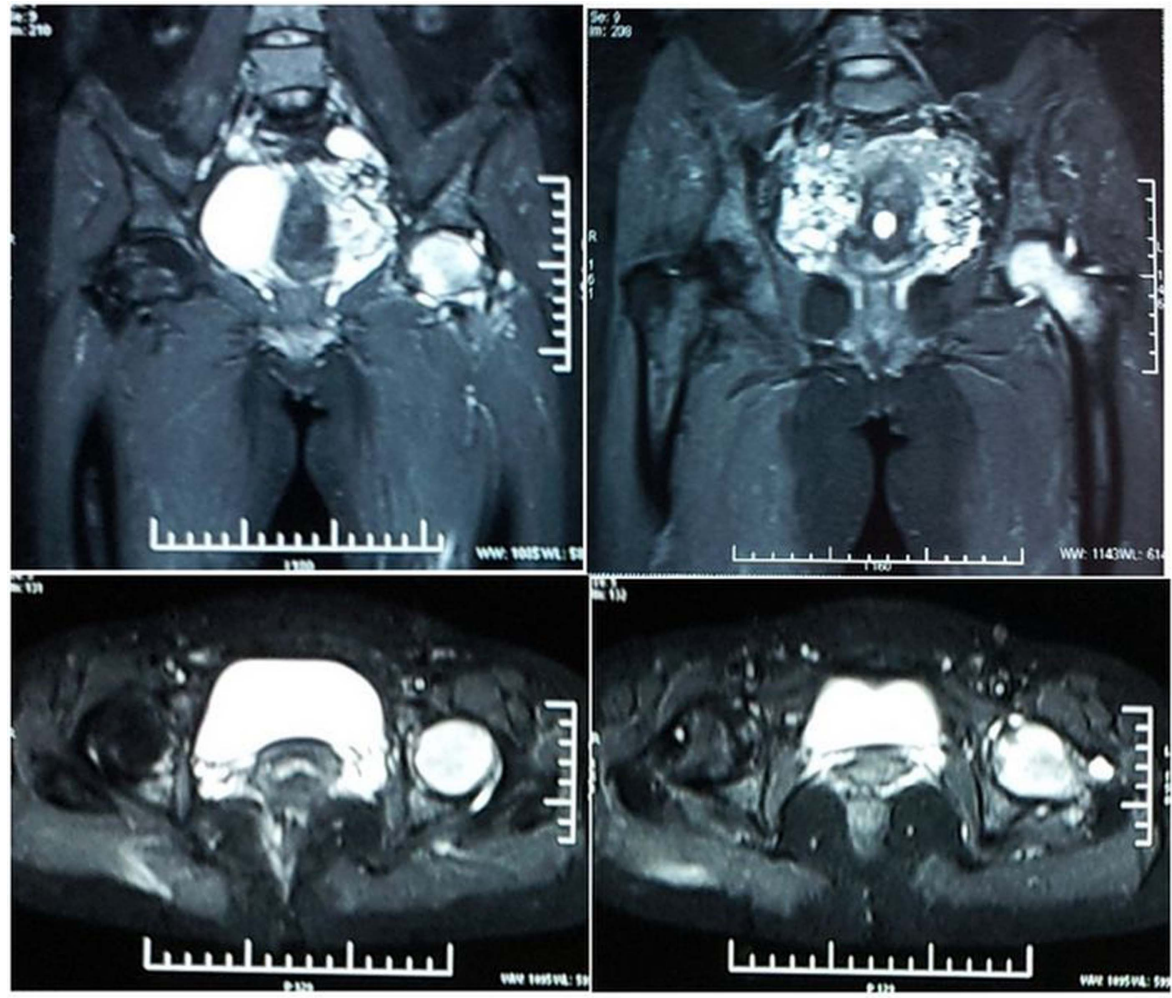

ischaemic insult affects only the haematopoietic and fatty elements. ${ }^{12}$ However, in avascular necrosis of the hip, cell death includes osteocytes. These two entities can be distinguished by several clinical and radiographic findings. Transient osteoporosis of the hip is clinically presented as acute-onset pain, aggravated by weight bearing and relieved by resting. The range of motion is usually preserved with occasional terminal restriction of movements. However, in AVN, the pain is aggravated by weight bearing and not relieved by resting. Transient osteoporosis of the hip is a self-limiting condition, but avascular necrosis of the hip is a progressive condition leading to collapse of the femoral head. In transient osteoporosis of the hip and avascular necrosis of the hip, a plain radiograph shows changes in 3-8 weeks after the onset of symptoms. ${ }^{3}$ In transient osteoporosis of the hip there is diffuse homogeneous involvement of the proximal femur (head-neck) and sometimes it extends up to the intertrochanteric region, acetabulum, iliac wings and ischiopubic rami; but there is always normal joint space with no osseous erosion or subchondral collapse. ${ }^{3}{ }^{6}$ In AVN, the involvement is focal and non-homogeneous, with subchondral collapse and decreased joint space in advance conditions. MRI can detect transient osteoporosis of the hip as early as $48 \mathrm{~h}$ after onset, and is highly sensitive, showing bone marrow oedema: low signal intensity of bone marrow on T1-weighted sequences and high signal intensity of bone marrow on fluid-sensitive (T2-weighted and fat suppressed) sequences. ${ }^{3}$ The oedema affects the subchondral bone of the femoral head and can extend towards the neck and intertrochanteric region. However, it is very difficult to differentiate it from the early stage of AVN with marrow oedema. The absence of band pattern, focal defects and subchondral changes on MRI is highly suggestive of transient osteoporosis of the hip. ${ }^{12-14}$ Transient osteoporosis of the hip is self-limiting and, within in time (6-8 months), the pain as well as the radiological abnormalities disappear spontaneously. ${ }^{36}$ It is important to identify this process and differentiate it from osteonecrosis so that early core decompression is not mistakenly carried out in transient osteoporosis. Transient osteoporosis of the hip is generally treated using conservative methods such as protected weight bearing, anti-inflammatory drugs and active and passive range of motion exercises.
Figure $4 \quad X$-rays of the pelvis with both hips (anteroposterior and lateral views) showing osteoporosis of the left femoral head.
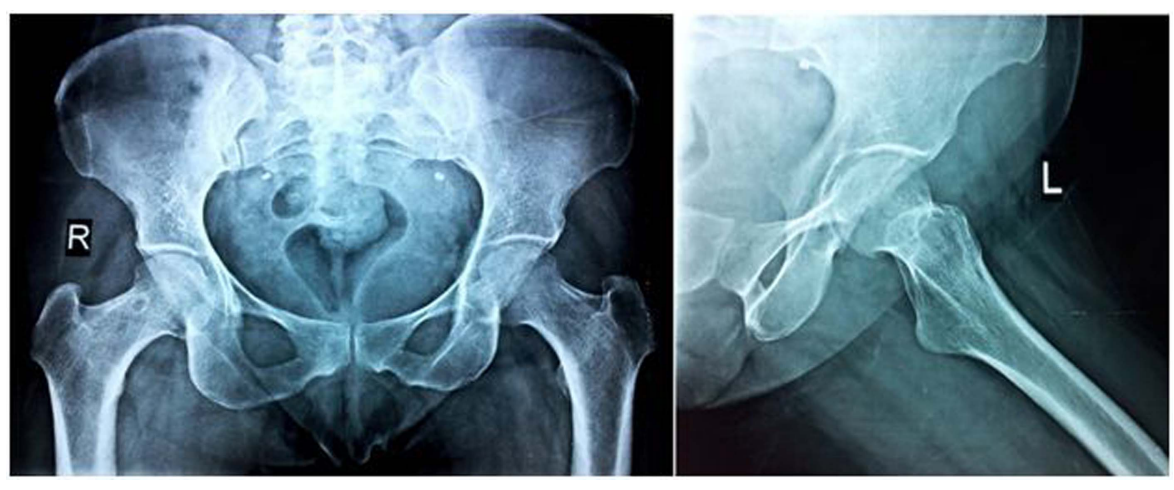
Only pregnancy is a risk factor for transient osteoporosis of the hip in women. Ours was a rare case of a 38-year-old nonpregnant woman with transient osteoporosis of the hip, managed conservatively leading to full recovery.

\section{Learning points}

- Transient osteoporosis of the hip is a very rare disorder in non-pregnant woman.

- It is important to identify this process and differentiate it, especially from osteonecrosis, so that early core decompression is not mistakenly carried out for transient osteoporosis of the hip.

- Conservative treatment is all that is needed and yields full recovery within 6 months.

Contributors JP, KBR, RK and AS helped in conceptualisation and formulation of the manuscript, collection of data, and final proof reading of the manuscript.

Competing interests None declared.

Patient consent Obtained.

Provenance and peer review Not commissioned; externally peer reviewed.

\section{REFERENCES}

1 Canale ST, Beaty JH. Campbell's operative orthopaedics. 12th edn, 2008. 1:139.

2 Schapira D. Transient osteoporosis of the hip. Semin Arthritis Rheum 1992;22:98-105.

3 Sandro C. Current concepts in magnetic resonance imaging of the adult hip and pelvis. Semin Roentgenol 2000;35:231-48.

4 Hayes CW, Conway WF, Daniel WW. MR imaging of bone marrow edema pattern: transient osteoporosis, transient bone marrow edema syndrome, or osteonecrosis. Radiographics 1993;13:1001-11.

5 Lakhanpal S, Ginsburg WW, Luthra HS, et al. Transient regional osteoporosis. A study of 56 cases and review of the literature. Ann Intern Med 1987;106:444-50.

6 Erdem HR, ÖzdeMırel AE, Nacır B. Transient osteoporosis of the hip in a non-pregnant woman: a case report. Turk J Phys Med Rehabil 2013;59:157-60.

7 Curtiss PH, Kincaid WE. Transient demineralization of the hip in pregnancy: a report of three cases. J Bone Joint Surg 1959;41(A):1327-33.

8 Hofmann S, Kramer J, Vakil-Adli A, et al. Painful bone marrow edema of the knee: differential diagnosis and therapeutic concepts. Orthop Clin N Am 2004;35:321-33.

9 Rishi V, Wahi P, Mahajan A. Transient osteoporosis of hip (migratory). JK Science 2008;10:194-6.

10 Uzun $\mathrm{M}$, Ayhan E, Beksac B, et al. Regional migratory osteoporosis and transient osteoporosis of the hip: are they all the same? Clin Rheumatol 2013;32:919-23.

11 Ma FY, Falkenberg M. Case reports: transient osteoporosis of the hip: an atypical case. Clin Orthop Relat Res 2006;445:245-9.

12 Yamamoto T, Kubo T, Hirasawa Y, et al. A clinicopathologic study of transient osteoporosis of the hip. Skeletal Radiol 1999;28:621-7.

13 Daniel WW, Sanders PC, Alarcon GS. The early diagnosis of transient osteoporosis by magnetic resonance imaging. A case report. J Bone Joint Surg Am 1992;74:1262-4.

14 Malizos KN, Zibis AH, Dailiana Z, et al. MR imaging findings in transient osteoporosis of the hip. Eur I Radiol 2004;50:238-44.

Copyright 2015 BMJ Publishing Group. All rights reserved. For permission to reuse any of this content visit http://group.bmi.com/group/rights-licensing/permissions.

BMJ Case Report Fellows may re-use this article for personal use and teaching without any further permission.

Become a Fellow of BMJ Case Reports today and you can:

- Submit as many cases as you like

- Enjoy fast sympathetic peer review and rapid publication of accepted articles

- Access all the published articles

- Re-use any of the published material for personal use and teaching without further permission

For information on Institutional Fellowships contact consortiasales@bmjgroup.com

Visit casereports.bmj.com for more articles like this and to become a Fellow 